HUNGARIAN AGRICULTURAL ENGINEERING

$N^{\circ} 28 / 2015$ 5-10

Published online: http://hae-journals.org/

HU ISSN 0864-7410 (Print) / HU ISSN 2415-9751(Online)

DOI: 10.17676/HAE.2015.28.5
PERIODICAL OF THE COMITTEE OF AGRICULTURAL AND BIOSYSTEM ENGINEERING OF

THE HUNGARIAN ACADEMY OF SCIENCES and

SZENT ISTVÁN UNIVERSITY

Faculty of Mechanical Engineering

\title{
CONDITIONS OF USING PROPELLER STIRRING IN BIOGAS REACTORS
}

\author{
Author(s): \\ Z. Bártfai - I. Oldal - L. Tóth - I. Szabó - J. Beke
}

Affiliation:

Szent István University, Faculty of Mechanical Engineering, 1.Páter K. street. Gödöllő, H2100

Email address:

bartfai.zoltan@gek.szie.hu, toth.laszo@gek.szie.hu, oldal.istvan@gek.szie.hu, szabo.istvan@gek.szie.hu, beke.janos@gek.szie.hu

\begin{abstract}
Preparing and further cutting of bovine dung which also contains straw, and of corn silage happens in special cutters, from which it is fed into the pre-fermentation tank. The primary mixing of liquid manure, and sewage water, including other fluids happens in a heated pre-container. The foodstuffs which are their expiration dates, and various other waste (household waste, greasy materials from washing, depleted frying oil, fats from grease-traps, etc.) are accepted in the pounder. This is where the cutting and separation of wrappings of boxed products happens as well, after which the thin parts are fed into an autoclave, and kept at $70 \mathrm{oC}$ for at least four hours to sterilise them.

The profit-oriented biogas plants consider the static, calculable production of electric and heat energy a fundamental goal, which requires the gas production to be continuous and free of hindrances [1]. In case of these factories, the gas yield for time unit may be lowered by various operation factors, but mainly malfunctions (errors, the foam within fermentation tanks from time to time, etc.). Incorrectly mixing or homogenising the materials arriving in the fermentation tanks also counts as a hindering operation factor. Our work details the basic questions of how the stirring inside fermentation tanks happens physically.
\end{abstract}

\section{Keywords}

biogas, stirring attributes, propeller fans, fermentation tanks

\section{Introduction}

Requirements for the stirring system are as follows:

- The density can be set for the entire mass even after mixing more dense, or more thin materials.

- Constant temperature and $\mathrm{pH}$ values can be achieved for the entire volume.

- The heat can be transferred from heating surfaces, and can be equalised.

- The micro-organisms are in a forced interaction with the nutrients.

- The entire volume is used, there are no so-called "dead areas".

- The hindering material parts are thinned.

- The subsidence of the substrate is prevented, and the nutrient content is homogenised.
- The bacteria release gas into the mass, which then reaches its surface in bubble form due to the so-called velocity shear of the material masses shifting compared to each other due to stirring.

Research analyses' conducted in biogas plants conclude that the homogenisation of materials fed into the fermentation tanks is not sufficient. Defining efficiency isn't simple due to the number of parameters which have an effect on fermentation. The physical constitution of materials, the level of cutting, the size of individual grains, the length of individual strands in materials which contain f.e. straw, etc. all have to be evaluated. The chemical constitution of materials, the contents of the materials f.e. $\mathrm{C} / \mathrm{N}$ ratios, $\mathrm{pH}$ values, dry material contents, etc., their inhibitor content, etc. all have to be calculated. Kamarad et al. [2] believes that most of the energy supplied is used for stirring, which is dependent on the viscosity of the material. The quality of stirring also determines the intensity of gas yield. During the stirring, they used a hydroxide-monohydrate solvent as a tracing indicator, and checked its quantity in various areas. The method proved to be feasible. In case of high concentrations, the fluid behaved as a non-Newton fluid. The indicator method helped to verify the usefulness of CFD (Computational Fluid Dynamics) modelling. Below are contour graphs of bladed stirring fans for their velocity (a) in [m/s], and viscosity (b) in [Pas] (Figure 1).

International literature discusses the stirring process inside biogas reactors in great length $[3,4]$. Various small samples were constructed for the goal of modelling the process, using simpler, cheaper methods. The main problem of these small samples is that the physical form of the material is hard to match with the system, and a model mass close to the original is hard to create. In order to raise reliability, the processes are simulated both in small scale, and close to real values, using various simulation software. These modelling processes are plagued by the problem of following the viscosity, and the susceptibility to fraction of materials. Therefore, they are riddled with errors. CFD modelling is considered to be one of the most effective method for determining main attributes $[5,6]$.

The small sample and the CFD method together are sufficient for solving basic problems in their entirety, f.e. identifying the dead areas which form during the stirring process, determining the velocity shear values, and the overall visual simulation of the stirring [7]. We can also make a guess on the energetic implementation, since calculating the hydraulic resistance of 
various stirring machines is possible as well [8]. Hydraulic resistance has a significant impact on energy consumption, which becomes substantial in case of big tanks. Another problem is the factory safety situation in case of some stirring methods. This factor is defined by two perspectives: the possible errors in the system, and the technological problems, meaning the fact that various materials stick to the stirring machine's surface; and to defend against and minimise the materials chipping the machinery away. During the stirring of various materials, various behaviours can be seen, as materials which contain strands are different to materials which contain heavy grains, the second of which includes both sand and small pebbles.

Nowadays' most widely used stirring methods can be seen on Figure 2.

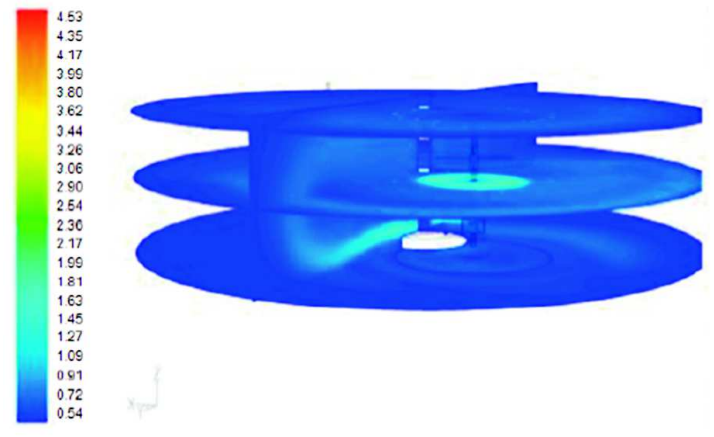

a.

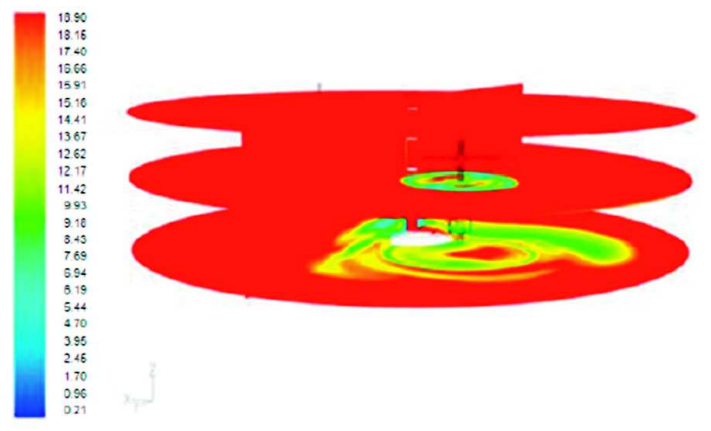

b.

Figure 1 . The velocity of mass for vertical bladed stirring fans (a) in [m/s], and viscosity contours in [Pas] inside a $300 \mathrm{~m}^{3} \operatorname{tank}[2]$

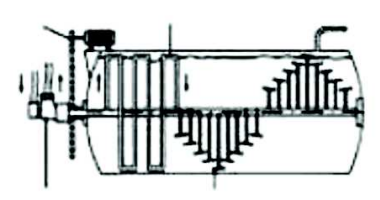

A

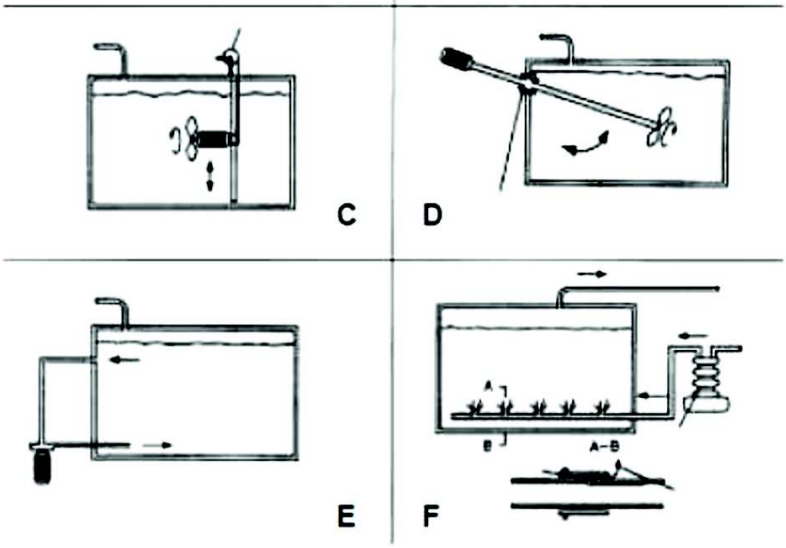

Figure 2. Most common stirring variations

A) Horizontal blade mixer B) Vertical blade mixer C) Variable height / flow propeller D) Propeller installed on a rocker

E) Hydraulic stirring F) Air injection stirring

International literature shows that the propeller fan may be the perspectivical one of the various stirring methods, since it has the least hydraulic resistance, its surface can be made the least susceptible to materials' sticking, and the velocity of outbound mass flows can be changed to a significant decree by setting the RPM of the engines. The engines and connection units manufactured with an isolation similar to diving-pumps can be set to different angles within the stirring space, moreover, software programs can be made to conduct this task as well. According to what's been said until now, a universal stirring method does not exist. The actual setting and operations of the stirring machine are determined by the physical and chemical attributes of the materials fed into the tank, and the shape and size of the stirring space are also something to be considered. To satisfy requirements (consistent temperature, physical and chemical attributes in the entire space, and prevention of divergence) knowing the material's momentary attributes is important, for which a software-controlled RPM and rotation direction can be chosen. Various experiments also tried the combination of stirring elements (Figure 3 ).

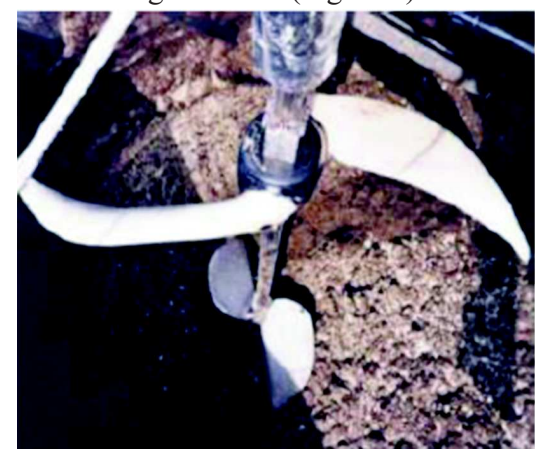

Figure 3. Combination of vertical bladed and propeller stirring fans [5]

Figure 4 illustrates the most common stirring element solutions found in literature.

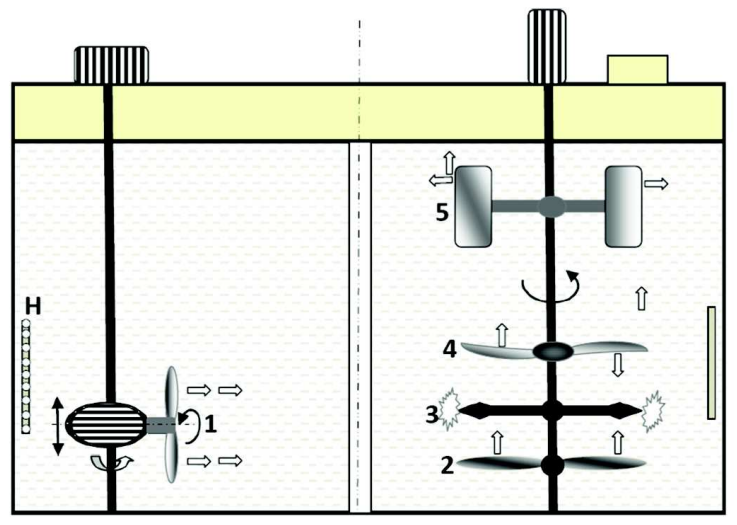

Figure 4. Modelled forms most common in literature. 1 - some propeller axial (possible to set height, direction and RPM), and vertical axis solution variants: 2 - large propeller delivering upwards, 3 - simple turbulent blade, 4 - wing blade,

5 - bladed propeller delivering upwards and sideways 
This research considers the propeller stirring method the most efficient, hence we analysed this one. The most common solutions among propeller stirrers were the variable position ones (Figures 5 and 6).

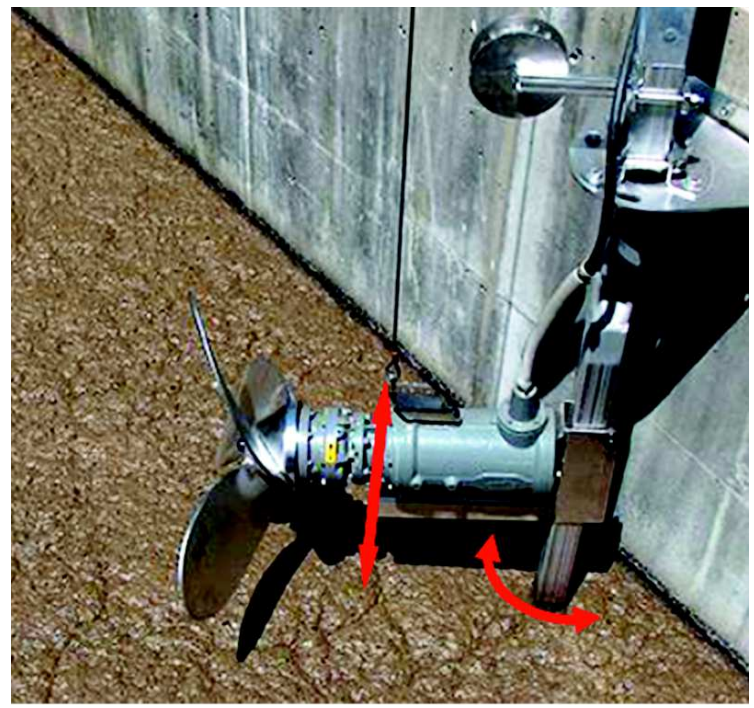

Figure 5. Changing height and direction

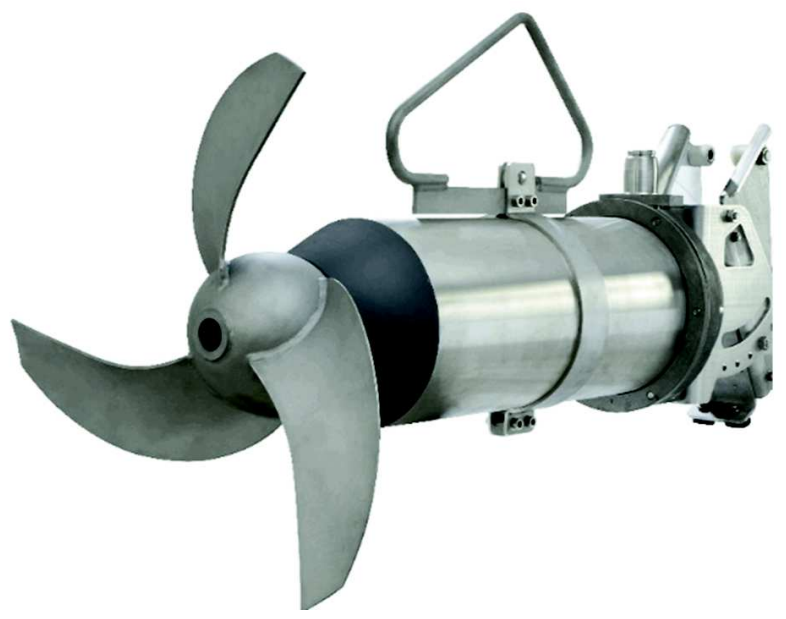

A

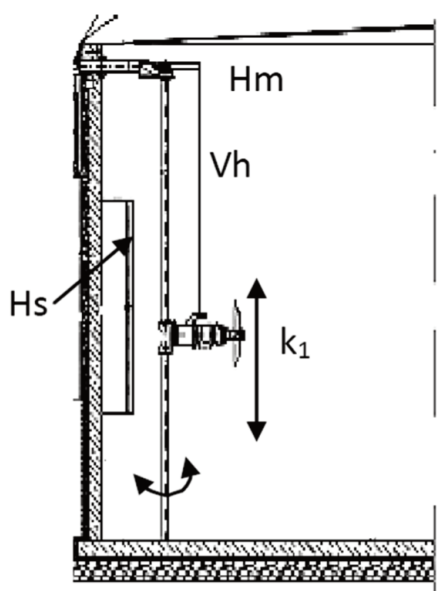

B

Figure 6. Propeller stirrers A - propeller and engine, $\mathrm{k}_{1}$ - installing the variable stirring fan, $\mathrm{Vh}$ - vertical setting, $\mathrm{Hm}$ - Horizontal setting, $\mathrm{Hs}$ - heat pipes

\section{Material and methods}

\section{Continuity in case of axial rowing}

We evaluated and modelled the stream circumstances of the propeller stirring method considered to be the most efficient by our source literature. The modelling and its results are introduced in the research of Bártfai and his colleagues $[9,10]$.

- The theoretic equations of the flowing liquid are based on the following assumptions:

- flow in the entire stream tube is stationary,

- the fluid can't be compressed (its density - p - is static),

-the fluid is frictionless,

-we disregard the gravitational field,

-axial speed is constant in each sections,

- radial velocity is zero.

The basis for defining the continuity equation is Figure 7.

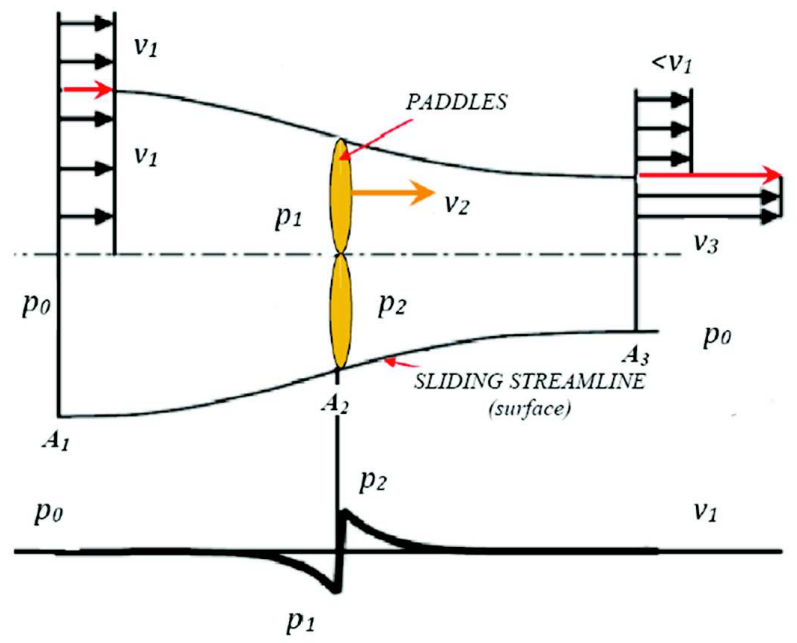

Figure 7. Section curve of the symmetrical current surface bordering the paddles and the medium flowing through their area

Based on these, the equation of continuity:

$$
\dot{m} \rho A v=\dot{m} \rho A_{2} v_{2}=\dot{m} \rho A_{3} v_{3}=\text { holds true. }
$$

With the proper indexes, we can present the equation for each section of the stream tube.

A sudden increase in tangential velocity may be possible on the border-streamline (the so-called sliding streamline). The liquid enters the stream tube on the left with a $\mathrm{v}_{1}$ relative speed (we can also say that the mixer is advancing towards the left with $\mathrm{a}-\mathrm{v}_{1}$ velocity in the freestream). The flow speed of the liquid inside the stream tube and outside of it is equal in this section (see the Figure). The medium gains speed compared to the mixing blades inside the stream tube, and leaves on the right side with a higher $\left(v_{3}>v_{1}\right)$ velocity, while the relative velocity outside the stream tube is static $\left(\mathrm{v}_{1}\right)$. Similarly, pressure is also constant $\mathrm{p}_{0}$, and while it gains from $\mathrm{p}_{1}$ to $\mathrm{p}_{2}$ on the agitator, leaving the stream tube, it recedes to $\mathrm{p}_{0}$ again.

The thrust signifies the strength of the flow reaction, which presented from the pressure difference is as follows:

$$
F=\left(p_{2}-p_{1}\right) A_{2}
$$

Bernoulli equations before and after the mixer in the stream tube are as follows:

$$
\begin{aligned}
& p_{0}+\frac{\rho}{2} v_{1}^{2}=p_{1}+\frac{\rho}{2} v_{2}^{2} \\
& p_{2}+\frac{\rho}{2} v_{2}^{2}=p_{0}+\frac{\rho}{2} v_{3}^{2}
\end{aligned}
$$


After defining $\mathrm{p}_{1}$ and $\mathrm{p}_{2}$, and substituting them into the thrust:

$$
F=\frac{\rho}{2}\left(v_{3}^{2}-v_{1}^{2}\right) A_{2}=\rho \frac{v_{3}+v_{1}}{2}\left(v_{3}-v_{1}\right) A_{2}
$$

The impulse differences between entering and leaving fluids can only be caused by the force of the propeller blade, impacting the fluid.

$$
\dot{m} v_{1}+F-\dot{m} v_{3}=0
$$

Therefore, force $(\mathrm{F})$ is as follows:

$$
\dot{m}\left(v_{3}-v_{1}\right)=\rho A_{2} v_{2}\left(v_{3}-v_{1}\right)=\boldsymbol{F}=\rho A_{2} \frac{v_{3}+v_{1}}{2}\left(v_{3}-v_{1}\right)
$$

An important provision must be declared - the mass flow in the disk's section can be calculated using the given value for any sections of the stream tube:

$$
\dot{m}=A_{2} v_{2}
$$

If we substitute this into the equation, then after reduction, it looks as follows:

$$
v_{2}=\frac{v_{1}+v_{3}}{2}
$$

Substituting this into the equation of continuity:

$$
\frac{1}{A_{2}}=\frac{1}{2}\left(\frac{1}{A_{1}}+\frac{1}{A_{3}}\right)
$$

The increase in velocity inside the stream tube is as follows:

$$
\Delta v=v_{3}-v_{1}
$$

Meaning the value of $\mathrm{v}_{2}$ is as follows:

$$
v_{2}=v_{1}+\frac{\Delta v}{2}
$$

Hydraulic power is the product of mass flow and change in movement energy value by unit:

$$
P_{h y}=\dot{m}\left(\frac{v_{3}^{2}}{2}-\frac{v_{1}^{2}}{2}\right)=\dot{m} \Delta v v_{1}\left(1+\frac{\Delta v}{2 v_{1}}\right)
$$

The power consumed for mass transport is the product of velocity before the mixer $\left(\mathrm{v}_{1}\right)$ and the movement force $(\mathrm{F})$ :

$$
P_{f e}=v_{1} F=v_{1} \dot{m}\left(v_{3}-v_{2}\right)=v_{1} \dot{m} \Delta v
$$

Resulting in efficiency, which is as follows:

$$
\eta=\frac{P_{f e}}{P_{h y}}=\frac{1}{1+\frac{\Delta v}{2 v_{1}}}
$$

Compared to propeller blades, the difference is that the medium is far before the mixer, which makes the $\mathrm{v}_{1}$ velocity zero, also meaning that $\mathrm{A}_{1}$ cross section has to be infinite.

Efficiency can usually be $50-65 \%$, which may be further decreased by other mechanical losses.

Usually, the quotient of the axial velocity of the material flowing along the edges of the blades, and the rotation velocity of the blade's end point is almost the same as the tangent of the blade's angle. Therefore, the value which describes this would be:

$$
v_{2}=\operatorname{tg} \alpha D n
$$

In case of parallel and continuous flow of the fluid layers, the internal friction force $(\mathrm{F})$ counter to the direction of movement is proportional to the areas (A) of the surfaces in friction (moving on top of each other), and the velocity gradient(du/dy).

The proportion factor is the constant value defining the fluid's material quality, in other words, dynamic viscosity $(\eta=P a s)$ :

$$
\eta=\frac{A_{f}}{F_{s}} \frac{d v_{2}}{d y_{n}}
$$

Where: $\mathrm{v}_{2}-$ velocity,

$$
\mathrm{y}_{\mathrm{n}}-\text { thickness of the fluid layer }
$$

The shear stress can be defined by the physical values of F/A (force/area):

$\tau,\left[\mathrm{N} / \mathrm{m}^{2}\right]:$

$$
\tau=-\eta \frac{d v_{2}}{d y_{n}}
$$

Which makes the velocity gradient, in other words, the shear velocity $\left[\mathrm{s}^{-1}\right]$ :

$$
\dot{\gamma}=\frac{d v_{2}}{d y_{n}}
$$

Newton's viscosity law states that the shear stress between layers is proportional with the velocity gradient. This doesn't hold true for non-Newton liquids, for which the formula is more complex between the shear stress and the velocity gradient.

Dynamic viscosity $(\eta)$ can be defined as the quotient of the shear stress $\left(\tau=\mathrm{N} / \mathrm{m}^{2}\right)$ and the shear velocity $\left[\mathrm{kg} \mathrm{m}^{-1} \mathrm{~s}^{-1}\right.$, meaning $\mathrm{Pa}$ s] as follows:

$$
\eta=-\frac{\tau}{\dot{\gamma}}
$$

This means the dynamic viscosity within fluids depends on the shear stress, and the velocity gradient.

If the transport direction of stirring blades is contrary to each other, and they are set in different heights from the ground, the formula can't be defined with functions, which means using modelling which is suitable to displaying the phenomenon happening is required.

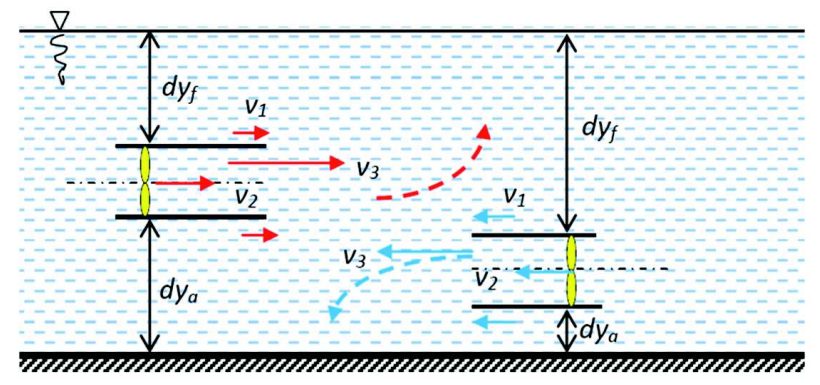

Figure 8. Two counter-rotating blades' interaction results in upwards and downwards streams. $\mathrm{dy}_{\mathrm{f}}-$ distance from the surface, $\mathrm{dy}_{\mathrm{a}}=$ distance from the ground, $\mathrm{v}_{1}$ - flow velocity of the fluid inside the fluid body, $\mathrm{v}_{2}$ - velocity of the fluid leaving the blades, $v_{3}-$ velocity of the fluid inside the fluid body, after it left the blades.

Due to this, the axial velocity value of the material flowing on the blade's edge differs in reality, which is why we can consider a $\mathrm{C}$ constant instead of the $\operatorname{tg} \alpha$ value, which is dependent on the stirring solution (surface area, angle of the blades, etc.), the shape and size of the tank, and the distance between fans and the walls of the tank. The dynamic viscosity of the material is also determined by the Reynolds number of the location (after all, the entire calculation holds true for a single construction and angle), meaning:

$$
v_{2}=C D n
$$

This makes mass flow:

$$
\dot{m}=\rho A_{2} v_{2}=C \rho D^{2} D n
$$

And the added (effective) energy:

$$
P_{h o}=C \rho D^{5} n^{3}
$$

The value of $\mathrm{C}$ is inversely proportional for the Reynolds value, but in case the Re value is high, it may become constant 
depending on the sizes of the tank. The dissipation of the 'fluid beam' exiting the propeller can be defined the same way as free fluid streams. Taking this, and our prior statements into consideration, modelling can be used to analyse the stirring of reactors from a fluid mechanics perspective, which does take the other elements of the system into consideration. F.e. we can define endless variations if we take any settings of three variable height and direction propeller stirring fans into consideration within a reactor. This is further complicated if their RPM can be varied without set levels. This amount of variations makes it easy to understand, how modelling and simulations are required. These methods may be used to select close-to-best favourable solutions, and choose the ones which seem the most favourable among them to conduct validation experiments on small samples or currently operating machines. The flow attributes which result from changes can be clearly seen on CDF models. As an example, see Figures 9 and 10 and 11.
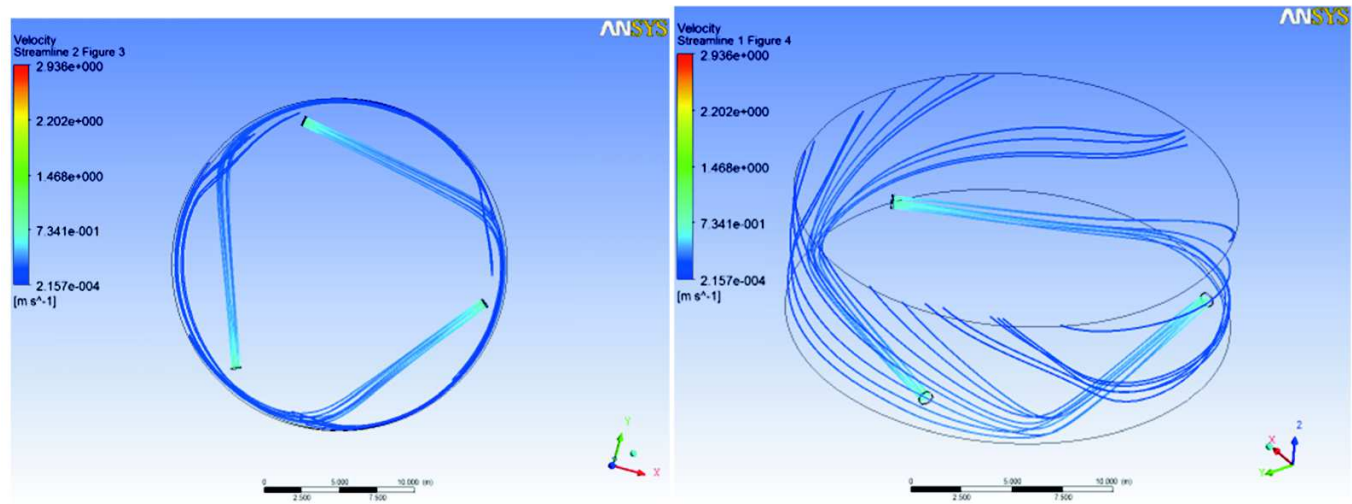

Figure 9. Three stirring fans positioned close to the ground, at the same height, towards the same flow directions.

The figures only show the flow expanding after the fans, which dissipates when it reaches the wall, and since the fans are close to the ground, the flows near the walls can only point downwards and dissipate. The middle section of the fermentation tanks doesn't house substantial movement at all.
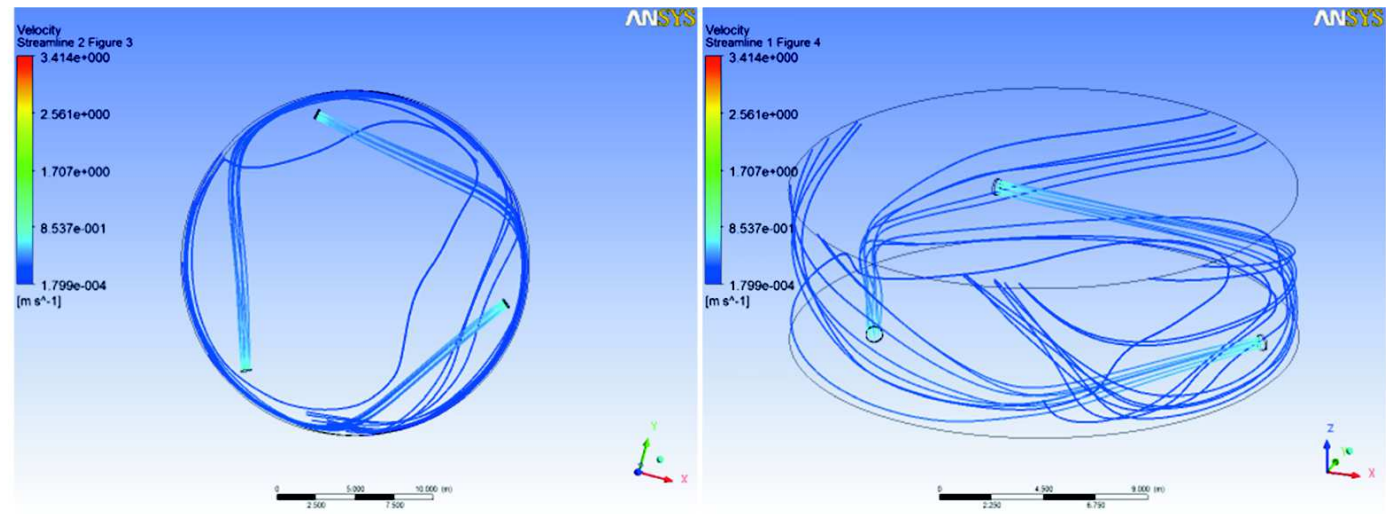

Figure 10. Three stirring fans positioned at different heights from the ground, towards the same flow directions.

This model also shows the flow expanding after the propeller, only dissipating when reaching the walls, but since two fans are farther from the ground, the dissipating flows can be seen flowing both downwards and upwards. Due to this, turbulent elements can also be seen near the walls.
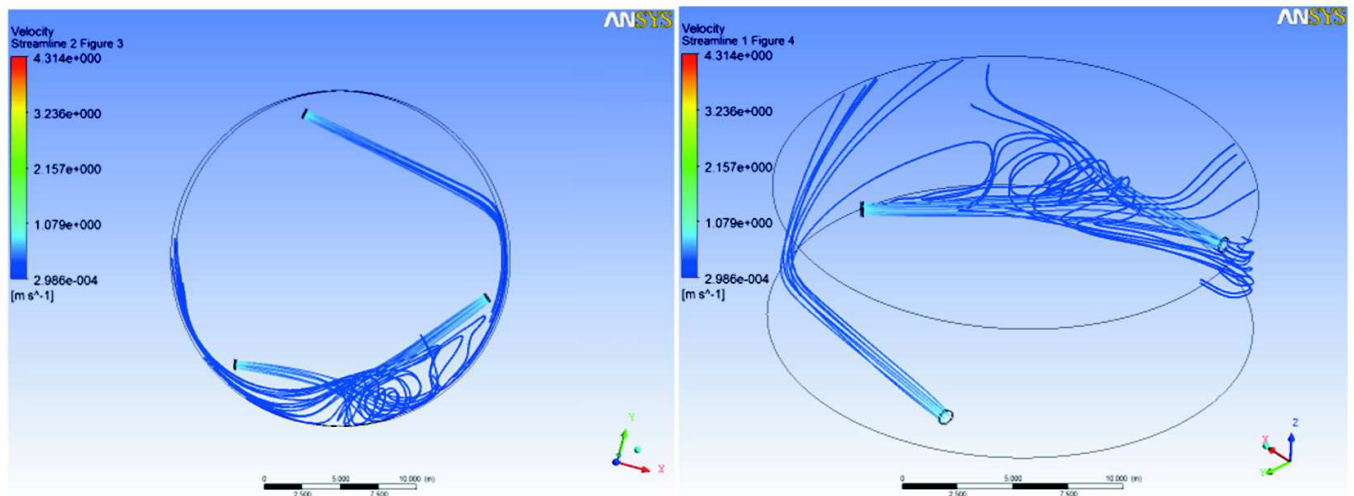

Figure 11. Three stirring fans positioned close to the ground, at the same height; two of them have identical flow directions, while the third has a different flow direction. 
The two stirring fans cause intensive turbulence (mass flow), but a third of the wall surface doesn't have any flows whatsoever.

The examples on the figures show how incorrect settings may cause technological errors, which is a detrimental effect on the efficiency of gas yield.

\section{Conclusion}

- The logic of the fermentation tank-system dictates that feeding the materials should be followed by a more intensive stirring due to the faster heat transport of materials inside and inbound, which can be conducted via increasing the RPM of the stirring fans, in other words, the transport mass flow. However, keeping the increased intensity up for a longer time is not a sound decision, since the increase of shear velocity between material molecules within the fermentation zones causes the life expectancy of methanogen bacteria to decrease.

-Also, a more intensive stirring phase also aids the shorter homogenisation of the material mass. Applying a frequency changer to the stirring fans to achieve this effect would be desirable, since it would make mass flows close to optimal, and a more intensive stirring phase achievable.

- Heat collection during the winter season can only be realised with a higher mass flow for current heat expelling surfaces.

- Masses which aren't moved can develop due to the incorrectly set stirring fans, which makes the validation of favourable settings determined during the modelling process a necessity (f.e. via inserting heat sensors).

- The length, frequency and intensity of stirring cycles can only be validated via evaluating gas yield.

- The increase in dynamic viscosity causes the time required for stirring to increase, which comes with increased energy consumption, most notably when the substrate contains an abundance of higher solidity, inorganic grains. This is why in the case of sewage waste, increasing the efficiency of the transport firms' sand separators is required.

-The optimum of stirring frequency also depends on the material, which is why determining it can only happen via empirically evaluating gas yield.

During our research, we took a look on stirring systems used in biogas reactors, and conducted the calculations for the propeller stirring fan system, which is considered to be the most variable. These calculations define the kinematic effects on the material. The direction, height and RPM - in other words, transport performance - may have to be changed in case of propeller stirring fan systems to achieve results better than any other stirring methods. This statement was supported partially by analyses made public in Issue 2, 2015 of HAE [9]. The setups resulting in the most favourable gas yields can be adapted to the changing conditions (the mass' biological, chemical and physical attributes) the best, and are the most approachable.

\section{Acknowledgement}

The study was supported by TÁMOP 4.2.1.C-14/1/KONV-20150002 .

\section{References}

[1.] Tóth L., Beke J., Bártfai Z., Szabó I., Hartdégen G., Oldal I., Blahunk Z.: 2014. Technological Features of Biogas Plants Using Mixed Materials, Hungarian Agricultural Engineering, $N^{\circ}$ 26/2014 39-46, Published online: HU ISSN 0864-7410 (Print) / HU ISSN 2415-9751(Online) http://dx.doi.org/10.17676/HAE.2014.26.39

[2.] Kamarad L., Pohn S., Bochmann G., Harasek M.: 2013. Determination of mixing quality in Biogas plant digesters using tracer Tests and computational fluid dynamics Cfd Acta Universitatis Agriculturae et Silviculturae Mendelianae Brunensis Volume LXI, 2013140 Number 5 http://dx.doi.org/10.11118/actaun201361051269

[3.] Schön M.: 2009. Numerical Modelling of Anaerobic Digestion Processes in Agricultural Biogas Plants, Dissertation, Eingereicht an der Leopold-Franzens-Universität Innsbruck, Fakultät für Bauingenieurwissenschaften zur Erlangung des akademischen Grades "Doktor Der Technischen Wissenschaften“, Innsbruck, Februar 2009 [4.] Bridgeman J.: 2012. Computational fluid dynamics modelling of sewage sludge mixing in an anaerobic digester Advances in Engineering Software Volume 44, Issue 1, February 2012, Pages 54-62

http://dx.doi.org/10.1016/j.advengsoft.2011.05.037

[5.] Maier C., Schlerka M., Weichselbaum W., Harasek M.: 2010. Development of agitation systems in biogas plants: Investigation of mixing characteristics, improvement of energy efficiency and scale-up using CFD, Chemical Engineering Transactions, Volume 21 Issue 1 Pages 1195-1200. ISSN 1974-9791. http://dx.doi.org/10.3303/CET1021200 [6.] Deglon D. A., Meyer C. J.: 2006. CFD modelling of stirred tanks: Numerical considerations, Minerals Engineering, Volume 19, p 1059-1068.

http://dx.doi.org/10.1016/j.mineng.2006.04.001

[7.] Liang Yu, Jingwei Ma, Shulin Chen.: 2012. Numerical simulation of mechanical mixing in high solid anaerobic digester, Bioresource Technology, Volume 102, Issue 2, January 2011, Pages 1012-1018

http://dx.doi.org/10.1016/j.biortech.2010.09.079

[8.] Terashima M., Goel R., Komatsu K., Yasui H., Takahashi H., Li Y. Y.: 2009. CFD simulation of mixing in anaerobic digesters, Bioresource Technology Volume 100, Issue 7, April 2009, Pages 2228-2233

http://dx.doi.org/10.1016/j.biortech.2008.07.069

[9.] Z. Bártfai, L. Tóth., I. Oldal, I. Szabó., J. Beke, N. Schrempf.: 2015, Modelling The Stirring Process of Biogas Plants Using Mixed Materials , HUNGARIAN AGRICULTURAL ENGINEERING, N 27/2015 Volume 1. In print.

[10.] Karaeva J. V., Khalitova G. R., Kovalev D. A., Trakhunova I. A.: 2015. Study of the Process of Hydraulic Mixing in Anaerobic Digester of Biogas Plant Chemical and Process Engineering. Volume 36, Issue 1, Pages 101-112, ISSN (Online) 2300-1925, http://dx.doi.org/10.1515/cpe-2015-0008 